\title{
Orbits of new Hipparcos binaries. II ${ }^{\star \star \star}$
}

\author{
I. I. Balega ${ }^{1}$, Y. Y. Balega ${ }^{1}$, K.-H. Hofmann ${ }^{2}$, E. V. Malogolovets ${ }^{1}$, D. Schertl ${ }^{2}$, \\ Z. U. Shkhagosheva ${ }^{1}$, and G. Weigelt ${ }^{2}$
}

\author{
1 Special Astrophysical Observatory, N.Arkhyz, Zelenchuk region, 369167 Karachai-Cherkesia, Russia \\ e-mail: balega@sao.ru \\ 2 Max-Planck-Institut für Radioastronomie, Auf dem Hügel 69, 53121 Bonn, Germany
}

Received 21 August 2005 / Accepted 24 October 2005

\section{ABSTRACT}

We continue the presentation of new orbits for Hipparcos binaries determined from regular speckle interferometric observations. Most of the data were collected in the period between 1998.77 and 2004.82 using the 6 m BTA telescope of the Special Astrophysical Observatory in Zelenchuk. New orbits are presented for six pairs: HIP 4809, HIP 4849, HIP 5531, HIP 19206, HIP 105947, and HIP 114922. One of the pairs, HIP 114922, has M dwarf components orbiting with a period of 19.72 yr. Two binaries, HIP 4809 and HIP 5531, have luminosity class IV components. The periods of the orbits range from $7.30 \mathrm{yr}$ to $28.99 \mathrm{yr}$. All of the orbits can be considered definitive; however, the total mass error for the systems remains high, mainly due to Hipparcos parallax error.

Key words. stars: binaries: visual - stars: fundamental parameters - stars: late-type

\section{Introduction}

This is the second in a series of publications presenting the orbits of new binary stars discovered during the Hipparcos mission (ESA 1997). Over 500 measurements of 180 new Hipparcos pairs were made between 1998.77 and 2004.82 with the speckle interferometer at the $6 \mathrm{~m}$ BTA telescope of the Special Astrophysical Observatory in Zelenchuk, Russia. The study focused primarily on nearby pairs of spectral types from late $\mathrm{F}$ to $\mathrm{M}$. The relative astrometry for our volumelimited sample of Hipparcos binary stars is given in the first papers of this series (Balega et al. 2002, 2004, 2005, hereafter Papers I, II, and III). A fraction of these systems display clear Keplerian motion of more than $20-30^{\circ} / \mathrm{yr}$ in position angle $\theta$ and/or 10-20 mas/yr in separation $\rho$. Due to the high precision of speckle observations, typically $0.5^{\circ}$ in $\theta$ and 1.5 mas in $\rho$, the data are sufficient for calculating first orbits for about 20 such systems. In addition to the relative positions, the obtained magnitude differences become important clues for deriving the physical properties of the components.

In the preceding paper (Balega et al. 2005, hereafter Paper IV), 6 orbits were given for $\mathrm{G}$ to $\mathrm{M}$ type Hipparcos pairs at distances of 15 to $66 \mathrm{pc}$ from the Sun. In this new paper, we report the results for 6 additional binaries of similar spectral types and distances. To derive the orbits, we used the already published speckle observations, supplemented with new

\footnotetext{
* Based on observations made with the 6-m BTA telescope, which is operated by the Special Astrophysical Observatory, Russia.

$\star \star$ Appendices A and B are only available in electronic form at http://www.edpsciences.org
}

measurements from the $6 \mathrm{~m}$ BTA telescope. For each pair, the data set consists of 9 to 15 independent measurements, including the first Hipparcos discovery.

\section{Observations and binary star orbits}

Our observational technique is described in Paper I of this series. Both the visual and infrared data from the $6 \mathrm{~m}$ telescope were used to define the orbits. The visual data were processed using the software developed at the Special Astrophysical Observatory, while the diffraction-limited image reconstructions in the infrared were performed at the Max-Planck-Institut fuer Radioastronomie in Bonn. Our speckle interferometric measurements made between 2001 and 2004 have not yet been published and are given here for those pairs under study for the first time.

The orbital elements were estimated using the Monet (1977) method, together with the differential correction of preliminary elements. All of the BTA observations were given unit weights. Horch et al. (1999, 2002) and Mason et al. (1999, 2001) measurements were taken with the weights 0.6 and 0.5 , respectively, following the aperture ratio of the telescopes, namely, 6:3,5 and 6:2,5. The Hipparcos relative positions for the epoch 1991.25 were given 0.5 weights; however, in two cases, HIP 5531 and 114922, the Hipparcos astrometric data were rejected because of large errors. The position angles $\theta$ were reduced to a common equinox 2000.0.

The results of our orbit calculations are presented in Table 1, where the first column gives the HIP number of the object, and the subsequent columns list, respectively, the 
Table 1. Orbital elements for six Hipparcos binaries.

\begin{tabular}{rrcccccccccrccccc}
\hline \hline HIP & \multicolumn{1}{c}{$\begin{array}{c}P \\
(\mathrm{yr})\end{array}$} & $\sigma_{P}$ & $T$ & $\sigma_{T}$ & $e$ & $\sigma_{\mathrm{e}}$ & $\begin{array}{c}a \\
(\mathrm{mas})\end{array}$ & $\sigma_{a}$ & $\begin{array}{r}i \\
(\mathrm{deg})\end{array}$ & $\sigma_{i}$ & \multicolumn{1}{c}{$\begin{array}{c}\sigma_{\Omega} \\
(\mathrm{deg})\end{array}$} & $\begin{array}{c}\omega \\
(\mathrm{deg})\end{array}$ & $\begin{array}{c}\sigma_{\omega} \\
\sigma_{\Theta} \\
(\mathrm{deg})\end{array}$ & $\begin{array}{c}\sigma_{\rho} \\
(\mathrm{mas})\end{array}$ \\
\hline 4809 & 16.41 & 0.11 & 1998.62 & 0.02 & 0.393 & 0.012 & 128 & 2 & 58.4 & 0.5 & 57.1 & 0.8 & 106.9 & 0.6 & 0.8 & 2 \\
4849 & 28.99 & 0.58 & 2002.68 & 0.01 & 0.678 & 0.005 & 465 & 7 & 144.3 & 0.8 & 92.6 & 2.2 & 205.4 & 1.9 & 1.0 & 3 \\
5531 & 7.30 & 0.11 & 1995.71 & 0.11 & 0.720 & 0.008 & 87 & 2 & 54.7 & 1.5 & 151.6 & 1.3 & 219.1 & 1.4 & 1.4 & 2 \\
19206 & 21.33 & 0.44 & 1996.77 & 0.08 & 0.686 & 0.011 & 223 & 6 & 122.2 & 1.2 & 38.2 & 1.7 & 255.6 & 1.1 & 0.9 & 2 \\
105947 & 18.79 & 0.57 & 1993.96 & 0.40 & 0.354 & 0.010 & 168 & 3 & 57.1 & 2.1 & 149.1 & 1.5 & 129.7 & 6.4 & 0.3 & 1 \\
114922 & 19.72 & 0.16 & 1999.81 & 0.02 & 0.440 & 0.003 & 219 & 2 & 117.1 & 0.3 & 28.6 & 0.5 & 324.8 & 0.7 & 0.3 & 1 \\
\hline
\end{tabular}

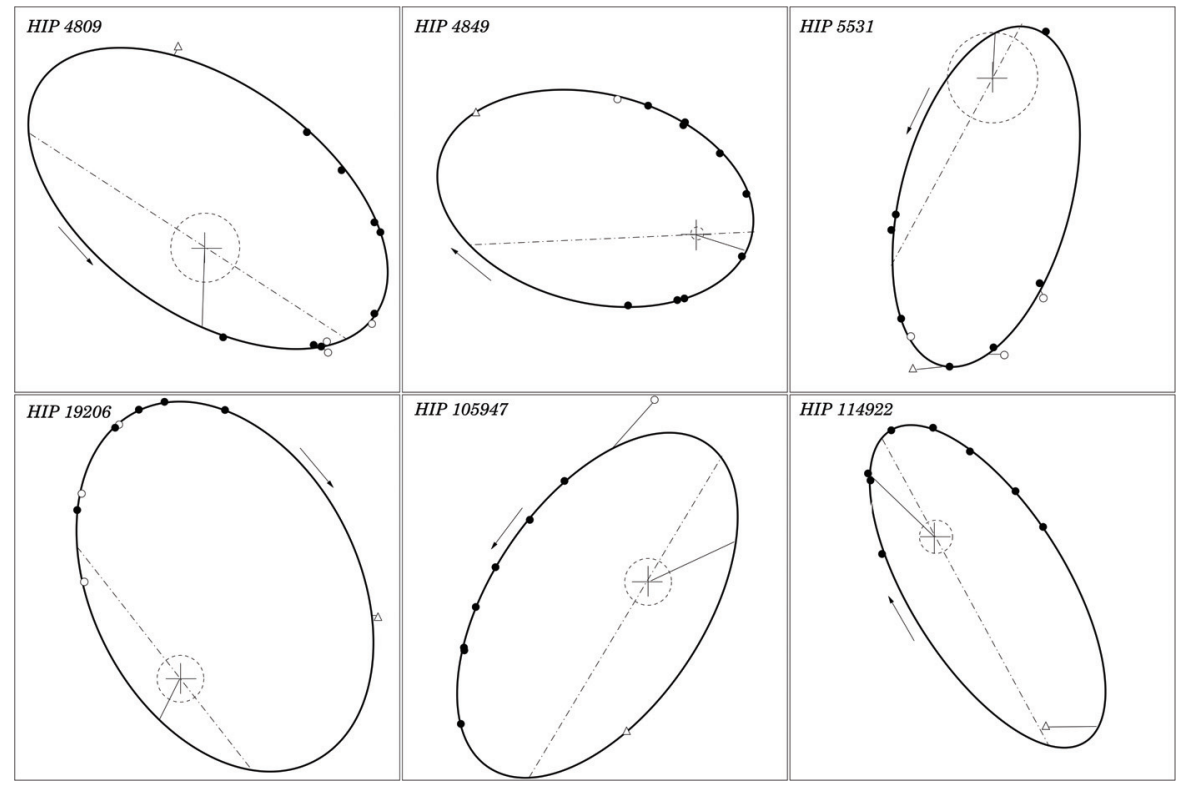

Fig. 1. Apparent ellipses representing the orbital elements for HIP 4809, HIP 4849, HIP 5531, HIP 19206, HIP 105947, and HIP 114922. The BTA speckle data are indicated by filled circles, other interferometric data by open circles, and Hipparcos first measurements by triangles. Residual vectors for all measures are plotted, but in most cases, they are smaller than the points themselves. The orbital motion direction is indicated by an arrow. The solid line shows the periastron position, while the dot-dashed line represents the line of nodes. North is up and east is to the left. The dashed circle at the position of the brighter component has a radius of $0.02^{\prime \prime}$. period $P$ (in years), periastron passage $T$, eccentricity $e$, semimajor axis $a$ of the true orbit (in milliarcseconds), orbit inclination $i$ (in degrees), position angle of the node $\Omega$ (in degrees, refers to the epoch 2000.0), and the longitude of periastron $\omega$ (in degrees), together with their formal errors. The last two columns give the rms deviation of $\theta$ and $\rho$ measurements from a solution that excludes the high-residual observations mentioned in the next section.

Following the Worley \& Heintz (1983) grading scheme (orbital coverage, separation residuals, no large gaps in position angle measurements), the presented orbits can be evaluated as grade 2, "good" (most of a revolution, well-observed, and with sufficient curvature of the motion) or grade 3, "reliable" (at least half of the orbit well defined). The orbits of HIP 105947 and HIP 114922 are the best of these 6 systems, while the HIP 4849 orbit shows the largest deviations. Tables A.1 to A.6 of the Appendix A summarize the speckle observations and the residuals of position angles $\theta$ and separations $\rho$ from the determined orbital elements. The first observations were taken from the Hipparcos Catalogue (ESA 1997).

The projection of the new set of orbits on the plane of the sky is presented in Fig. 1, in which our speckle measurements are plotted as filled circles, while other speckle measurements are shown as open circles. The Hipparcos 1991.25 discoveries are indicated by triangles.

In the late 1990's, optical and infrared speckle interferometry became an effective technique for measuring accurate brightness ratios for binary and multiple stars. We decided to include the existing magnitude difference measurements for our six systems in this paper. Table B.1 of Appendix B lists $30 \Delta m$ values obtained with the $6 \mathrm{~m}$ BTA telescope, together with 14 measurements appearing in the literature. The six 1991.25 measurements are the Hipparcos first observations ( $H p$ filter, $\lambda / \Delta \lambda=476 / 210 \mathrm{~nm}$ ). Not one of the objects is noted as potentially variable in the Hipparcos Catalogue.

Table 2 lists the derived basic physical properties and the main orbital parameters of the observed systems, including the 6 new Hipparcos binaries from Paper IV. For convenience, we have included the Hipparcos parallaxes and their standard deviations in Cols. 2 and 3 of the table. Column 4 gives the absolute magnitudes for the components derived from the mean speckle interferometric magnitude differences, total $V$ magnitudes of the systems from the SIMBAD database, and $\pi_{\mathrm{hp}}$. For these nearby stars, we assumed no absorption $\left(A_{\mathrm{V}}=0\right)$. Column 5 presents the corresponding spectral types for the luminosity class V stars. The exceptions are HIP 4809 and 5531, which have luminosity class IV components. For them, the spectral types were inferred from their color indices. Columns 6 to 10 give the periods of the systems in years, semi-major axes in AU, total masses from Kepler's third law, and standard deviations of the total masses found as

$\frac{\sigma_{\mathcal{M}}}{\mathcal{M}}=\left[9\left(\frac{\sigma_{a}}{a}\right)^{2}+4\left(\frac{\sigma_{P}}{P}\right)^{2}+9\left(\frac{\sigma_{\pi}}{\pi}\right)^{2}\right]^{1 / 2}$. 
Table 2. Main parameters of the orbits, properties of the components, and total masses for 12 new Hipparcos binaries. Six pairs with new orbits from Paper IV are also included.

\begin{tabular}{|c|c|c|c|c|c|c|c|c|c|c|c|}
\hline \multirow[t]{2}{*}{ HIP } & \multirow{2}{*}{$\begin{array}{c}\pi_{\mathrm{hp}} \\
\text { mas }\end{array}$} & \multirow{2}{*}{$\begin{array}{c}\sigma_{\pi} \\
\text { mas }\end{array}$} & \multicolumn{2}{|c|}{$\overline{M_{V}}$} & \multicolumn{2}{|c|}{$\overline{S p p}$} & \multirow{2}{*}{$\begin{array}{l}P \\
\mathrm{yr}\end{array}$} & \multirow{2}{*}{$\begin{array}{c}a \\
\mathrm{AU}\end{array}$} & \multirow{2}{*}{$\begin{array}{c}\mathcal{M} \\
\mathcal{M}_{\odot}\end{array}$} & \multirow{2}{*}{$\begin{array}{l}\sigma_{\mathcal{M}} \\
\mathcal{M}_{\odot}\end{array}$} & \multirow{2}{*}{$\begin{array}{c}\sigma_{\mathcal{M}} / \mathcal{M} \\
\%\end{array}$} \\
\hline & & & A & B & & B & & & & & \\
\hline 4809 & 13.94 & 0.90 & 3.1 & 3.3 & G6-C & G9IV & 16.41 & 9.18 & 2.90 & 0.57 & 20 \\
\hline 4849 & 46.61 & 1.61 & 6.7 & 8.4 & K3 & K8 & 28.99 & 9.77 & 1.17 & 0.14 & 12 \\
\hline 5531 & 17.41 & 0.67 & 3.2 & 4.0 & G0I & & 7.30 & 5.00 & 2.33 & 0.30 & 13 \\
\hline 11352 & 23.19 & 1.21 & 5.5 & 5.7 & G8 & G9 & 6.85 & 4.31 & 1.71 & 0.27 & 16 \\
\hline 14075 & 15.17 & 1.44 & 5.5 & 5.5 & G8 & G8 & 13.89 & 7.07 & 2.03 & 0.58 & 29 \\
\hline 14230 & 29.62 & 1.09 & 4.7 & 6.4 & $\mathrm{G} 2$ & K2 & 5.91 & 3.71 & 1.47 & 0.26 & 18 \\
\hline 14669 & 64.83 & 4.26 & 9.5 & 11.2 & M2 & M4 & 28.31 & 8.78 & 0.84 & 0.17 & 20 \\
\hline 19206 & 24.00 & 0.92 & 4.1 & 5.5 & G0 & G8 & 21.33 & 9.29 & 1.75 & 0.25 & 14 \\
\hline 105947 & 17.11 & 1.13 & 3.9 & 5.4 & F8 & G8 & 18.79 & 9.82 & 2.68 & 0.58 & 22 \\
\hline 106972 & 39.78 & 3.70 & 9.9 & 11.1 & M2 & M4 & 18.57 & 6.74 & 0.89 & 0.25 & 28 \\
\hline 111685 & 52.94 & 1.94 & 8.2 & 10.2 & K7 & M3 & 16.77 & 6.23 & 0.86 & 0.10 & 12 \\
\hline 114922 & 31.50 & 3.85 & 9.4 & 9.6 & M1 & M2 & 19.72 & 6.95 & 0.87 & 0.32 & 37 \\
\hline
\end{tabular}

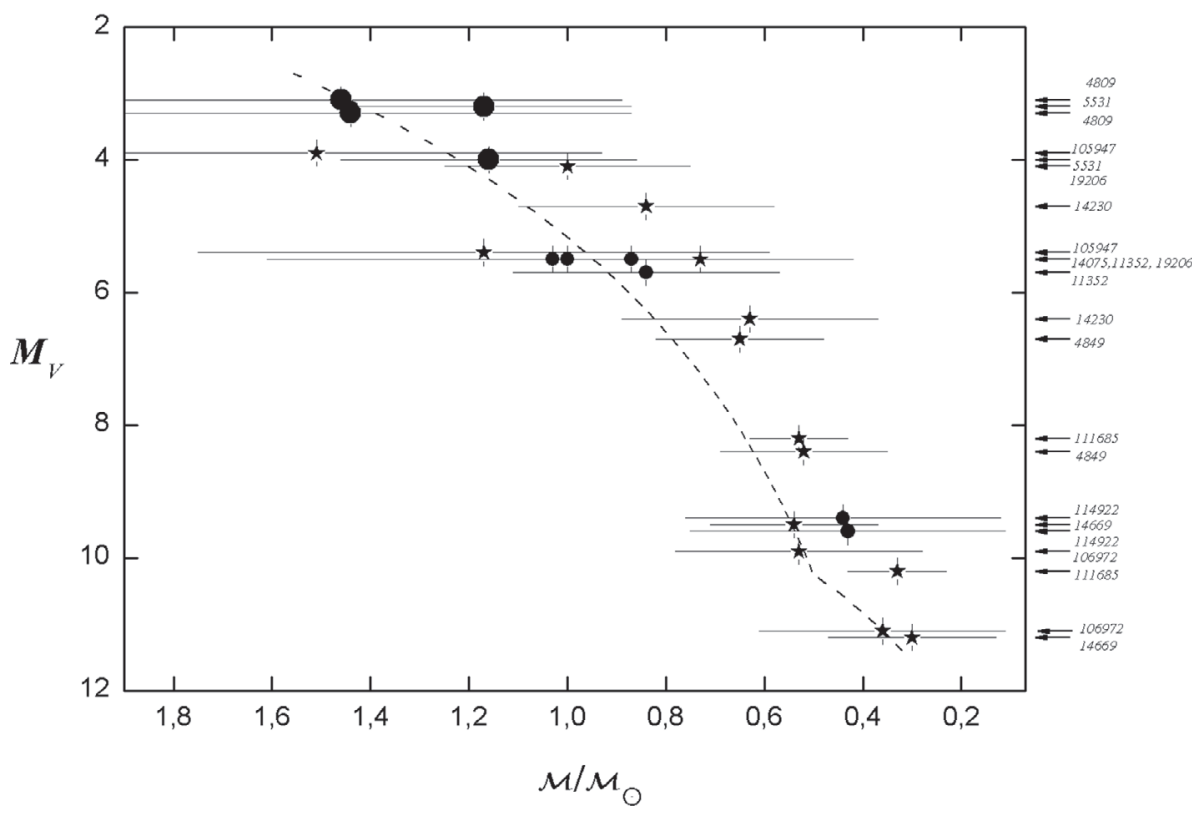

Fig. 2. Location of the components of 12 new Hipparcos binaries from Table 2 on the mass-luminosity diagram. Binaries with $\Delta m \leq 1$ are plotted as filled circles, while those with $\Delta m>1$ are plotted as stars. The larger circles indicate two systems with evolved components, HIP 4809 and HIP 5531. Error bars are taken the same as for the mass sums in Table 2. The dashed line represents the empirical $\mathcal{M} / L$ relation from Henry \& McCarthy (1993).

The last column in the table presents the total mass error in percent. Note that the semi-major axes of the orbits lie in the narrow range between 3.7 and $9.8 \mathrm{AU}$, which indicates the area of efficiency of speckle interferometry for the study of a nearby sample of Hipparcos binaries.

Comparison of $\sigma_{\mathcal{M}} / \mathcal{M}$ in Table 2 with the Hipparcos parallax relative error $\left(\sigma_{\pi} / \pi\right)_{\mathrm{hp}}$ leads to the conclusion that the masssum error is linearly increasing with $\left(\sigma_{\pi} / \pi\right)_{\mathrm{hp}}$ :

$\sigma_{\mathcal{M}} / \mathcal{M}=3\left[1+\left(\sigma_{\pi} / \pi\right)_{\mathrm{hp}}\right]($ per cent $)$.

Hence, with more precise parallaxes in the future (one may envision the GAIA astrometric mission), the quality of our orbits will be sufficient for deriving masses with an accuracy of about $5 \%$.

Figure 2 shows the location of the components of 12 binaries from Paper IV and the present study on the $\mathcal{M} / L$ diagram. To derive the mass ratios $q$ for all but two systems, we used the empirical mass-luminosity relation for main-sequence dwarfs from Henry \& McCarthy (1993), which is plotted as a dashed line in the figure. In the cases of HIP 5531 and 19206, we used the $q$ values from spectroscopic surveys (Nordström et al. 2004). The pairs with small magnitude differences are marked by filled circles, and those with $\Delta m>1$ mag are plotted as stars. The larger filled circles represent the evolved components of HIP 4809 and 5531.

The components of several binaries (HIP 19206, 14230, $4849,111685)$ are noticeably deviant from the $\mathcal{M} / L$ fits towards smaller masses. Note that all of them belong to systems with $\Delta m>1 \mathrm{mag}$. The difference between the locus of the $\mathcal{M} / L$ relation and our results can be explained by the different evolutionary stages and metallicities of the stars. However, because of the limited size of the sample, the effects of age and metallicity were not considered. The most probable reasons for the deviations are the mass-sum errors. A small change in the parallax value can shift the obtained mass sums towards the main region of the empirical $\mathcal{M} / L$ diagram. For example, a $\pi_{\mathrm{hp}}$ 
reduction from 24 mas to 22 mas will put the componets of HIP 19206 exactly on the $\mathcal{M} / L$ curve; the same is true if one takes $\pi=27$ mas in place of $\pi_{\mathrm{hp}}=30$ mas for HIP 14230 .

In Fig. 2, the mass-sum error bars for the binaries in the upper part of the diagram are significantly wider than for the low-mass pairs. This difference is explained by a smaller relative error of parallaxes for the nearby low-mass dwarfs.

For two systems from Paper IV, HIP 14669 and 106972, we obtained significantly larger dynamical parallaxes $\pi_{\mathrm{dn}}$ compared to the Hipparcos parallaxes, correspondingly, $\pi_{\mathrm{dn}}=$ 69.86 mas instead of $\pi_{\mathrm{hp}}=64.83$ mas and $\pi_{\mathrm{dn}}=44.85$ mas instead of $\pi_{\mathrm{hp}}=39.78$. Both of these systems have faint M-type components. Dynamical parallaxes for other pairs agree well with the Hipparcos parallaxes, giving a difference of 1-2 mas.

\section{Comments on individual systems}

\subsection{HIP $4809=H D 6009$}

The mean $\Delta m$ value from speckle and Hipparcos measurements, collected in Table B.1, is 0.14 mag in the $V$-band with a standard error of $0.03 \mathrm{mag}$. Here we supposed that $\Delta m$ does not change significantly in the range $\lambda 545-800 \mathrm{~nm}$. The only measurement in the $K$-band within the error gives the same value. With this magnitude difference in hand, we used the Hipparcos parallax value $\pi_{\mathrm{hp}}=13.94 \pm 0.90$ mas and $V=6.71$ from the SIMBAD database to obtain absolute magnitudes for the components $M_{\mathrm{A}}=3.1$ and $M_{\mathrm{B}}=3.3$. Together with the color $(B-V)=0.783 \pm 0.007$ given in the Hipparcos cata$\log$, this corresponds to G6-G9 type subgiant stars. This estimate agrees with the luminosity class given by Yoss (1961) from his analysis of the strength of the $\lambda 4077$ ionized strontium line based on the prism spectra from the Curtis Schmidt telescope of the University of Michigan and slit spectrograms from the 60 inch reflector of the Mt. Wilson Observatory. The luminosity class IV for the components is also supported by the moderate cyanogen absorption at $\lambda 4216$.

The orbit was defined from 16 astrometric measurements, including the first resolution by Hipparcos, one observation by Mason et al. (2001) with the $2.1 \mathrm{~m}$ telescope of the McDonald Observatory (0.06" resolution limitation), two observations by Horch et al. (2002) with the $3.5 \mathrm{~m}$ WIYN telescope at Kitt Peak, and 12 observations with the $6 \mathrm{~m}$ BTA telescope. To obtain a consistent orbit, we had to adjust the quadrants of $\theta$ measurements from the $2.1 \mathrm{~m}$ and the $3.5 \mathrm{~m}$ telescopes. The quadrant of the first Hipparcos is also inconsistent with the majority of further speckle observations, which can be explained by the low value of the magnitude difference and the largerthan-typical error on the Hipparcos $\Delta H_{\mathrm{p}}$ magnitude. The mean value of residuals for the BTA speckle measurements is 0.4 mas in $\rho$ and $0.5^{\circ}$ in $\theta$. However, despite small residuals of the measurements, the total mass error for the system is $20 \%$, which is explained by the overwhelming input of the $\pi_{\mathrm{hp}}$ error.

\section{2. $H I P 4849=H D 6101=G J 3071$}

The total proper motion of this nearby system, listed in the Lowell Observatory survey as G $1-43$, is $\mu=0.41^{\prime \prime} / \mathrm{yr}$
(Giclas et al. 1961). Based on these data, Sandage \& Kowal (1986) included GJ 3071 in the list of possible new nearby subdwarfs. However, it was found that the measured $U-B=+0.86$ for $\mathrm{G} 1-43$ at the given $B-V=+1.01$ color does not deviate from the corresponding Hyades $U-B$ value, and, consequently, the star cannot be a subdwarf. In his compilation survey for stars that may be within 25 pc of the sun, Halliwell (1979) first mentioned the discordant photometric and trigonometric parallaxes for the star, which could be a sign of its binary nature.

After the first resolution of the system by Hipparcos in 1991.25, eleven speckle measurements covered about $2 / 3$ of the visual orbit. One speckle observation belongs to Mason et al. (2001), while all others are from the 6 m BTA telescope. With an orbital period of 29 years, this pair has the largest semi-major axis of the visual orbit among the binaries listed in Table 1.

\section{3. $H I P 5531=H D 6840$}

This is a high proper motion star with $\mu_{\alpha}=0.25^{\prime \prime} / \mathrm{yr}$. The common color index of the system $B-V=0.553 \pm 0.005$, taken from the SIMBAD database (the Hipparcos measurement), corresponds to a G0 spectral type. Chen et al. (2000), Lambert \& Reddy (2004), and Nordström et al. (2004) determined its effective temperature from the reddening-corrected $b-y, c_{1}$ and $m_{1}$ indices in the range $\log T_{\mathrm{e}}=3.7670-3.7679$ corresponding to an early G-dwarf. However, the photometric distance determination ( $\pi_{\mathrm{ph}}=37$ mas) is not in agreement with the Hipparcos parallax $\pi_{\mathrm{hp}}=17$, suggesting evolved components. From our mean magnitude difference in Table B.1, $\Delta m=0.68$, the absolute magnitudes of the stars in the pair are $M_{\mathrm{A}}=3.21$ and $M_{\mathrm{B}}=3.89$, respectively. The authors cited above find the metallicity for HIP 5531 in the range of $-0.62 \leq[\mathrm{Fe} / \mathrm{H}] \leq-0.44$. Given the $T_{\mathrm{e}}, M_{V}$, and $[\mathrm{Fe} / \mathrm{H}]$ for the star, an age of $7 \mathrm{Gyr}$ is estimated for the main component by interpolation among the evolutionary tracks provided by Girardi et al. (2000).

Due to good phase coverage of the interferometric orbit, the period and semi-major axis of HIP 5531 are well-defined. Note that $2 / 3$ of its total mass error comes from the Hipparcos parallax error. Recently, HIP 5531 was recognized to be also a SB2 with a mass ratio $0.99 \pm 0.02$ (Nordström et al. 2004). With an accuracy of about $10 \%$, the subsequent individual masses of the components are $\mathcal{M}_{\mathrm{A}}=1.17 \mathcal{M}_{\odot}$ and $\mathcal{M}_{\mathrm{B}}=1.16 \mathcal{M}_{\odot}$. The next periastron passage for HIP 5531 will be in the beginning of 2010. To obtain more accurate masses and luminosities for the components, it is worth keeping the binary in the lists of future spectroscopic and interferometric monitorings.

\section{4. $H I P 19206=H D 26040$}

Spectral type G0V results from the Eggen (1985) and Nordström et al. (2004) photometry that gives $(b-v)=0.36$ and $\log T_{\mathrm{e}}=3.772$. This relates to the main component of the system because the secondary is 1.34 mag fainter, as follows from our Table B.1. For the secondary, our differential photometry leads to $M_{\mathrm{B}}=5.5$ and spectral type G8V. 
The system is also given as SB2 in the Nordström et al. (2004) survey of $\mathrm{F}$ and $\mathrm{G}$ dwarfs in the Solar neighborhood. The mass ratio $q=0.73 \pm 0.11$ from this paper fits well with our $q=0.80$ arising from the masses of dwarfs of the corresponding spectral types.

\section{5. $H I P 105947=H D 204236$}

The distribution of speckle observations along the apparent ellipse is uneven, with most measurements concentrated near the apastron. Therefore, following the criteria of Worley \& Heintz (1983), the orbit must be graded only as "reliable". The Mason et al. (1999) speckle point of 1997.7 falls well outside the apparent ellipse after assigning the right quadrant; hence, it was ignored in the calculations.

\section{6. $H I P 114922=$ GJ 893.4}

This high proper-motion star is also known as Vyss 860 . Absolute magnitudes for the components were calculated with the assumption that $\Delta m_{R}=0.2$. Apart from the first Hipparcos observation, all other measurements define a reliable ellipse of the visual orbit. Note that two speckle measurements were obtained with the $6 \mathrm{~m}$ telescope just at the moment of periastron passage in 1999.8. The Hipparcos parallax of this faint system is given with an accuracy of only $12.2 \%$. As a consequence, the mass-sum is defined with a standard error of $37 \%$ (input of the $\sigma_{\pi_{\mathrm{hp}}}$ error in the muss-sum error exceeds $99 \%$ ).

\section{Summary}

We have presented new speckle measurements of the angular separations $\rho$ and position angles $\theta$ of the components of 6 new Hipparcos binaries, and derive new orbits and masses based upon Hipparcos parallaxes and relative speckle astrometry obtained between 1997-2004. For all of the six systems, in Table B. 1 we present $\Delta m$ measurements from the $6 \mathrm{~m}$ telescope in the wavelength range of $\lambda=545 \mathrm{~nm}$ to $\lambda=2115 \mathrm{~nm}$. For comparison, the Hipparcos $\Delta V_{H \mathrm{p}}$ differences and Horch et al. (2004) $\Delta m$ measurements are also included. These differential magnitudes allow the absolute magnitudes and spectral types of the components to be determined.

The mass-sum accuracies for the 6 new systems range between 12 and $37 \%$. The dominant source of uncertainty in the overall error budget for the masses remains the parallax in all cases. For example, in the case of HIP $114922,99 \%$ of the total error comes from the $\pi_{\mathrm{hp}}$ error.

Two systems, HIP 4809 and 5531, have evolved components. In addition, HIP 5531 was recently found to be SB2 with a mass ratio of 0.99 (Nordström et al. 2004). Because of the even phase coverage of speckle observations and the relatively short orbital period, this pair has the most accurate speckle orbit among the studied systems. Another pair given as SB2 by Nordström et al. (2004) is HIP 19206. The physical parameters of these two binaries can be significantly improved when the spectroscopic orbits become available.

The final result is the obtained location of the components on the "mass-absolute magnitude" diagram. New Hipparcos binaries with orbits from Paper IV of this series are also plotted. Most of the dwarf systems fall close to the empirical relation of Henry \& McCarthy (1993), but with four "outliers" due to large relative mass-sum errors.

About half of the components in Table 2 have masses between 0.20 and $0.50 \mathcal{M}_{\odot}$. As mentioned by Henry et al. (1999), this region of the diagram is perhaps more poorly determined than the lower masses. They explain this peculiarity by the large scatter of the empirical data on the plateau in the $\mathcal{M} / L$ relation caused by the effects of hydrogen molecules in the atmospheres combined with the deep convective regions. In addition, substantial progress in defining the bottom of the $\mathcal{M} / L$ diagram has been made recently because of the special attention given to the study of brown dwarfs and planets in multiple systems. Our observational goal is to define the relative orbits for a few dozen new Hipparcos binaries with an accuracy of semimajor axes better than $1 \%$. New spectroscopic radial velocities and chemical compositions from the $6 \mathrm{~m}$ telescope will allow us to improve the orbital elements and the knowledge of the physical properties of the components.

Acknowledgements. The speckle interferometry program at the $6 \mathrm{~m}$ telescope was partly supported by the Russian Foundation for Basic Research through grant No. 04-02-17563a. This publication made use of the SIMBAD database, operated at the CDS in Strasbourg, France.

\section{References}

Balega, I. I., Balega, Y. Y., Hofmann, K.-H., et al. 2002, A\&A, 385, 87 (Paper I)

Balega, I. I., Balega, Y. Y., Maksimov, A. F., et al. 2004, A\&A, 422, 627 (Paper II)

Balega, I. I., Balega, Y. Y., Maksimov, A. F., et al. 2005, Bull. Special Astrophys. Obs., 59 (Paper III)

Balega, I. I., Balega, Y. Y., Hofmann, K.-H., et al. 2005, A\&A, 433, 591 (Paper IV)

Chen, Y. Q., Nissen, P. E., Zhao, G., et al. 2000, A\&AS, 141, 491

Eggen, O. J. 1985, PASP, 97, 807

ESA 1997, The Hipparcos and Tycho Catalogues, ESA SP-1200

Giclas, H. L., Burnham, R., \& Thomas, N. G. 1961, Lowell Obs. Bull., 5,61

Girardi, L., Bressan, A., Bertelli, G., \& Chiosi, C. 2000, A\&AS, 141, 371

Halliwell, M. J. 1979, ApJS, 41, 173

Henry, T. J., \& McCarthy, D. W. 1993, AJ, 106, 773

Henry, T. J., Franz, O. G., Wasserman, L. H., et al. 1999, ApJ, 512, 864

Horch, E. P., Ninkov, Z., van Altena, W. F., et al. 1999, AJ, 117, 548

Horch, E. P., Robinson, S. E., Meyer, R. D., et al. 2002, AJ, 123, 3442

Horch, E. P., Meyer, R. D., \& van Altena, W. F. 2004, AJ, 127, 1727

Lambert, D. L., \& Reddy, B. E. 2004, MNRAS, 349, 757

Mason, B. D., Martin, C., Hartkopf, W. I., et al. 1999, AJ, 117, 1890

Mason, B. D., Hartkopf, W. I., Holdenried, E. R., \& Rafferty, T. J. 2001, AJ, 121, 3224

Monet, D. G. 1977, ApJ, 214, L133

Nordström, B., Mayor, M., Andersen, J., et al. 2004, A\&A, 418, 989

Pluzhnik, E. A. 2005, A\&A, 431, 587

Sandage, A., \& Kowal, C. 1986, AJ, 91, 1140

Yoss, K. M. 1961, ApJ, 134, 809

Worley, C. E., \& Heintz, W. D. 1983, Publ. USNO, 24, part 7 


\section{Online Material}


I. I. Balega et al.: Orbits of new Hipparcos binaries. II., Online Material p 2

\section{Appendix A: Hipparcos and speckle relative} astrometry of six new binaries, and residuals

Table A.1. HIP 4809.

\begin{tabular}{crrrrl}
\hline \hline Epoch & \multicolumn{1}{c}{$\theta$} & \multicolumn{1}{c}{$\rho$} & \multicolumn{1}{c}{$\Delta \theta$} & \multicolumn{1}{c}{$\Delta \rho$} & \multicolumn{1}{c}{ Reference } \\
\hline 1991.250 & 8.0 & 0.118 & -1.5 & 0.004 & ESA (1997) \\
1998.772 & 189.5 & 0.050 & 0.1 & 0.000 & Paper I \\
1999.747 & 228.2 & 0.086 & -0.1 & -0.001 & Paper I \\
1999.813 & 229.5 & 0.088 & -0.3 & 0.000 & Paper II \\
1999.823 & 229.7 & 0.094 & -0.3 & 0.005 & Mason et al. (2001) \\
1999.888 & 232.5 & 0.089 & 1.1 & -0.002 & Horch et al. (2002) \\
2000.767 & 245.5 & 0.107 & -1.4 & 0.001 & Horch et al. (2002) \\
2000.873 & 249.2 & 0.106 & 0.7 & 0.000 & Paper III \\
2000.876 & 248.8 & 0.106 & 0.2 & 0.000 & Paper III \\
2001.753 & 261.9 & 0.107 & 0.2 & 0.000 & Paper III \\
2001.753 & 261.9 & 0.107 & 0.2 & 0.000 & Paper III \\
2001.753 & 262.2 & 0.107 & 0.5 & 0.000 & this paper \\
2001.845 & 263.1 & 0.107 & 0.0 & 0.000 & this paper \\
2002.726 & 275.2 & 0.103 & -2.0 & 0.002 & this paper \\
2002.796 & 278.6 & 0.100 & 0.2 & -0.001 & this paper \\
2003.928 & 299.9 & 0.092 & 0.3 & -0.001 & this paper \\
2004.815 & 318.7 & 0.090 & 0.6 & 0.000 & this paper \\
\hline
\end{tabular}

Table A.2. HIP 4849 .

\begin{tabular}{crcrrl}
\hline \hline Epoch & \multicolumn{1}{c}{$\theta$} & \multicolumn{1}{c}{$\rho$} & \multicolumn{1}{c}{$\Delta \theta$} & \multicolumn{1}{c}{$\Delta \rho$} & \multicolumn{1}{c}{ Reference } \\
\hline 1991.250 & 62.0 & 0.711 & -0.4 & 0.000 & ESA (1997) \\
1997.718 & 31.4 & 0.433 & -0.1 & -0.007 & Mason et al. (1999) \\
1998.772 & 21.4 & 0.376 & 0.1 & 0.003 & Paper I \\
1999.747 & 7.3 & 0.302 & -0.5 & -0.005 & Paper I \\
1999.813 & 6.6 & 0.305 & 0.1 & 0.003 & Paper II \\
2000.876 & 342.7 & 0.231 & 0.8 & 0.000 & Paper III \\
2001.753 & 307.8 & 0.182 & -0.6 & -0.001 & Paper III \\
2002.736 & 245.6 & 0.146 & -1.7 & 0.002 & this paper \\
2002.799 & 245.1 & 0.141 & 2.8 & -0.001 & this paper \\
2003.788 & 169.1 & 0.178 & -0.4 & 0.002 & this paper \\
2003.928 & 162.9 & 0.188 & 0.2 & 0.000 & this paper \\
2004.815 & 135.2 & 0.275 & -0.1 & -0.002 & this paper \\
\hline
\end{tabular}

Table A.3. HIP 5531.

\begin{tabular}{cccrrl}
\hline \hline Epoch & \multicolumn{1}{c}{$\theta$} & \multicolumn{1}{c}{$\Delta$} & \multicolumn{1}{c}{$\Delta \theta$} & \multicolumn{1}{c}{$\Delta \rho$} & \multicolumn{1}{c}{ Reference } \\
\hline 1991.250 & 165.0 & 0.136 & -4.6 & 0.004 & ESA (1997) \\
1997.630 & 162.8 & 0.122 & 1.5 & 0.001 & Horch et al. (1999) \\
1998.774 & 171.9 & 0.131 & 0.3 & -0.001 & Paper I \\
1999.634 & 182.6 & 0.125 & 3.4 & 0.001 & Horch et al. (2002) \\
1999.821 & 180.4 & 0.121 & -0.7 & 0.000 & Paper II \\
2000.765 & 193.3 & 0.102 & 0.6 & 0.004 & Horch et al. (2002) \\
2000.878 & 193.1 & 0.095 & -1.5 & 0.001 & Paper III \\
2002.796 & 310.4 & 0.032 & 0.4 & 0.001 & this paper \\
2003.788 & 145.0 & 0.075 & 1.2 & 0.002 & this paper \\
2003.928 & 146.8 & 0.082 & -0.4 & 0.000 & this paper \\
2004.816 & 159.5 & 0.116 & -0.6 & -0.003 & this paper \\
\hline
\end{tabular}

Table A.4. HIP 19206.

\begin{tabular}{rrcrrl}
\hline \hline Epoch & \multicolumn{1}{c}{$\theta$} & \multicolumn{1}{c}{$\rho$} & \multicolumn{1}{c}{$\Delta \theta$} & \multicolumn{1}{c}{$\Delta \rho$} & \multicolumn{1}{c}{ Reference } \\
\hline 1991.250 & 287.0 & 0.178 & -1.4 & 0.005 & ESA (1997) \\
1997.830 & 44.5 & 0.116 & -1.7 & 0.001 & Horch et al. (1999) \\
1998.772 & 31.2 & 0.169 & 0.2 & -0.001 & Paper I \\
1999.012 & 28.0 & 0.179 & -0.5 & -0.001 & Horch et al. (2002) \\
2000.759 & 13.5 & 0.224 & -1.2 & 0.002 & Horch et al. (2002) \\
2000.876 & 14.2 & 0.223 & 0.2 & -0.001 & Paper III \\
2001.753 & 8.5 & 0.234 & -0.1 & 0.001 & Paper III \\
2002.797 & 3.0 & 0.238 & 0.5 & 0.001 & this paper \\
2004.824 & 350.4 & 0.234 & -0.3 & 0.000 & this paper \\
\hline
\end{tabular}

Table A.5. HIP 105947.

\begin{tabular}{crcrrl}
\hline \hline Epoch & \multicolumn{1}{c}{$\theta$} & \multicolumn{1}{c}{$\rho$} & \multicolumn{1}{c}{$\Delta \theta$} & \multicolumn{1}{c}{$\Delta \rho$} & \multicolumn{1}{c}{ Reference } \\
\hline 1991.250 & 172.0 & 0.127 & 0.1 & -0.001 & ESA (1997) \\
1997.720 & 357.6 & 0.151 & -17.8 & 0.037 & Mason et al. (1999) \\
1998.780 & 39.2 & 0.108 & -0.4 & 0.001 & Paper I \\
1999.745 & 62.2 & 0.110 & 0.0 & -0.001 & Paper I \\
2000.864 & 84.6 & 0.127 & 0.1 & 0.001 & Paper III \\
2001.752 & 98.5 & 0.143 & 0.4 & 0.000 & Paper III \\
2002.799 & 110.0 & 0.163 & -0.4 & -0.001 & this paper \\
2002.799 & 110.7 & 0.163 & 0.2 & -0.001 & this paper \\
2004.815 & 127.5 & 0.195 & -0.1 & 0.000 & this paper \\
\hline
\end{tabular}


I. I. Balega et al.: Orbits of new Hipparcos binaries. II., Online Material p 3

Table A.6. HIP 114922 .

\begin{tabular}{crcrrl}
\hline \hline Epoch & \multicolumn{1}{c}{$\theta$} & \multicolumn{1}{c}{$\rho$} & \multicolumn{1}{c}{$\Delta \theta$} & \multicolumn{1}{c}{$\Delta \rho$} & Reference \\
\hline 1991.250 & 210.0 & 0.256 & -10.5 & -0.033 & ESA (1997) \\
1998.774 & 108.4 & 0.065 & 0.0 & 0.001 & Paper I \\
1999.742 & 48.7 & 0.099 & 0.0 & -0.003 & Paper I \\
1999.815 & 46.2 & 0.107 & -0.1 & 0.001 & Paper II \\
2000.864 & 21.9 & 0.134 & 0.5 & 0.000 & Paper III \\
2001.845 & 0.5 & 0.127 & -0.5 & 0.001 & this paper \\
2002.736 & 337.8 & 0.108 & 0.2 & -0.002 & this paper \\
2003.927 & 299.6 & 0.108 & 0.1 & 0.001 & this paper \\
2004.815 & 275.3 & 0.126 & -0.1 & 0.000 & this paper \\
2004.821 & 275.2 & 0.126 & -0.1 & 0.000 & this paper \\
\hline
\end{tabular}

\section{Appendix B: Magnitude differences for six new} Hipparcos binaries

Table B.1. Speckle interferometric magnitude differences and Hipparcos $\Delta H \mathrm{p}$ (HMA04 = Horch et al. 2004; P05 = Pluzhnik 2005).

\begin{tabular}{|c|c|c|c|c|}
\hline HIP & Date & $\Delta m$ & $\lambda / \Delta \lambda, \mathrm{nm}$ & Ref. \\
\hline \multirow[t]{11}{*}{4809} & 1991.25 & $0.28 \pm 0.41$ & $\overline{V_{H \mathrm{p}}}$ & ESA (1997) \\
\hline & 1998.77 & $0.17 \pm 0.15$ & $545 / 30$ & Paper I \\
\hline & 1998.77 & $0.10 \pm 0.24$ & $545 / 30$ & $\mathrm{P} 05$ \\
\hline & 1999.75 & $0.30 \pm 0.16$ & $2115 / 214$ & Paper I \\
\hline & 1999.81 & $0.19 \pm 0.04$ & $610 / 20$ & Paper II \\
\hline & 1999.89 & $0.05 \pm 0.15$ & $648 / 41$ & HMA04 \\
\hline & 2000.77 & $0.22 \pm 0.15$ & $503 / 40$ & HMA04 \\
\hline & 2000.87 & $0.12 \pm 0.19$ & $800 / 110$ & Paper III \\
\hline & 2000.87 & $0.16 \pm 0.05$ & $600 / 30$ & Paper III \\
\hline & 2001.75 & $0.00 \pm 0.12$ & $545 / 30$ & Paper III \\
\hline & 2001.75 & $0.00 \pm 0.12$ & $600 / 30$ & Paper III \\
\hline \multirow[t]{7}{*}{4849} & 1991.25 & $1.87 \pm 0.04$ & $V_{H \mathrm{p}}$ & ESA (1997) \\
\hline & 1998.77 & $1.72 \pm 0.04$ & $610 / 20$ & Paper I \\
\hline & 1999.75 & $0.78 \pm 0.12$ & $2115 / 214$ & Paper I \\
\hline & 1999.81 & $1.66 \pm 0.05$ & $610 / 20$ & Paper II \\
\hline & 2000.88 & $1.66 \pm 0.03$ & $600 / 30$ & Paper III \\
\hline & 2001.75 & $1.48 \pm 0.03$ & $600 / 30$ & Paper III \\
\hline & 2001.75 & $1.48 \pm 0.03$ & $600 / 30$ & Paper III \\
\hline \multirow[t]{8}{*}{5531} & 1991.25 & $0.47 \pm 0.24$ & $V_{H \mathrm{p}}$ & ESA (1997) \\
\hline & 1997.63 & $0.73 \pm 0.08$ & $648 / 41$ & HMA04 \\
\hline & 1998.77 & $0.71 \pm 0.03$ & $545 / 30$ & Paper I \\
\hline & 1998.77 & $0.73 \pm 0.03$ & $545 / 30$ & P05 \\
\hline & 1999.63 & $0.45 \pm 0.15$ & $601 / 42$ & HMA04 \\
\hline & 1999.82 & $0.75 \pm 0.03$ & $610 / 20$ & Paper II \\
\hline & 2000.76 & $0.88 \pm 0.08$ & $648 / 41$ & HMA04 \\
\hline & 2000.88 & $0.71 \pm 0.02$ & $545 / 30$ & Paper III \\
\hline \multirow[t]{7}{*}{19206} & 1991.25 & $1.32 \pm 0.24$ & $V_{H \mathrm{p}}$ & ESA (1997) \\
\hline & 1997.83 & $1.37 \pm 0.08$ & $648 / 41$ & HMA04 \\
\hline & 1998.77 & $1.42 \pm 0.04$ & $610 / 20$ & Paper I \\
\hline & 1999.01 & $1.33 \pm 0.08$ & $648 / 41$ & HMA04 \\
\hline & 2000.76 & $1.29 \pm 0.08$ & $648 / 41$ & HMA04 \\
\hline & 2000.88 & $1.39 \pm 0.02$ & $545 / 30$ & Paper III \\
\hline & 2001.75 & $1.26 \pm 0.02$ & $600 / 20$ & Paper III \\
\hline \multirow[t]{7}{*}{105947} & 1991.25 & $1.17 \pm 0.60$ & $V_{H \mathrm{p}}$ & ESA (1997) \\
\hline & 1998.78 & $1.50 \pm 0.06$ & $545 / 30$ & Paper I \\
\hline & 1998.78 & $1.50 \pm 0.04$ & $545 / 30$ & P05 \\
\hline & 1999.75 & $1.16 \pm 0.14$ & $2115 / 214$ & Paper I \\
\hline & 2000.86 & $1.40 \pm 0.02$ & $545 / 30$ & Paper III \\
\hline & 2001.75 & $1.29 \pm 0.05$ & $850 / 75$ & Paper III \\
\hline & 2001.75 & $1.31 \pm 0.06$ & $600 / 30$ & Paper III \\
\hline \multirow[t]{4}{*}{114922} & 1991.25 & $0.44 \pm 0.57$ & $V_{H \mathrm{p}}$ & ESA (1997) \\
\hline & 1998.77 & $0.00 \pm 0.35$ & $800 / 60$ & Paper I \\
\hline & 1999.82 & $0.16 \pm 0.09$ & $800 / 60$ & Paper II \\
\hline & 2000.86 & $0.00 \pm 0.23$ & $800 / 110$ & Paper III \\
\hline
\end{tabular}

